\title{
ON THE PEANO CURVE OF LEBESGUE*
}

\section{J. SCHOENBERG}

Let $f(t)$ be the even continuous function of period two which is defined in the interval $(0,1)$ as follows: $f(t)=0$ in $(0,1 / 3), f(t)=1$ in $(2 / 3,1)$, and $f(t)$ is linear in $(1 / 3,2 / 3)$. Our curve is defined by the parametric equations

$$
\begin{aligned}
& x(t)=\frac{1}{2} f(t)+\frac{1}{2^{2}} f\left(3^{2} t\right)+\frac{1}{2^{3}} f\left(3^{4} t\right)+\cdots, \\
& y(t)=\frac{1}{2} f(3 t)+\frac{1}{2^{2}} f\left(3^{3} t\right)+\frac{1}{2^{3}} f\left(3^{5} t\right)+\cdots, \quad 0 \leqq t \leqq 1 .
\end{aligned}
$$

The inequalities $0 \leqq f(t) \leqq 1$ imply $0 \leqq x(t) \leqq 1,0 \leqq y(t) \leqq 1$, as well as the uniform convergence of both series (1), and hence imply the continuity of $x(t), y(t)$. All there remains to show is that our curve will pass through an arbitrarily given point

(2) $x_{0}=\frac{a_{0}}{2}+\frac{a_{2}}{2^{2}}+\frac{a_{4}}{2^{3}}+\cdots, y_{0}=\frac{a_{1}}{2}+\frac{a_{3}}{2^{2}}+\frac{a_{5}}{2^{3}}+\cdots, a_{\nu}=0,1$,

of the square $0 \leqq x, y \leqq 1$, whose coordinates are given by their binary expansions. Indeed, let

$$
t_{0}=\frac{2 a_{0}}{3}+\frac{2 a_{1}}{3^{2}}+\frac{2 a_{2}}{3^{3}}+\cdots+\frac{2 a_{k-1}}{3^{k}}+\frac{2 a_{k}}{3^{k+1}}+\cdots .
$$

If $a_{0}=0$, we have $0 \leqq t_{0} \leqq 2 / 3^{2}+2 / 3^{3}+\cdots=1 / 3$, hence $f\left(t_{0}\right)=0$; if $a_{0}=1$, we have $2 / 3 \leqq t_{0} \leqq 2 / 3+1 / 3=1$, hence $f\left(t_{0}\right)=1$. In either case $f\left(t_{0}\right)=a_{0}$. Similarly $3^{k} t_{0}=$ even integer $+2 a_{k} / 3+2 a_{k+1} / 3^{2}+\cdots$ shows that

$$
f\left(3^{k} t_{0}\right)=a_{k}, \quad k=0,1,2, \cdots .
$$

Now (1), (2), and (4) imply $x\left(t_{0}\right)=x_{0}, y\left(t_{0}\right)=y_{0} . \dagger$

\section{Colby College}

* Presented to the Society, April 16, 1938.

$\dagger$ Lebesgue, Leçons sur l' Intégration, Paris, 1928, pp. 44-45, defines the functions $x(t), y(t)$, first on Cantor's ternary set $T$, of points $t_{0}$ of the form (3), by means of the equations (2). Having proved their continuity on $T$, Lebesgue extends their definition throughout $(0,1)$ by linear interpolation over each one of the denumerable set of open intervals of which the set complementary to $T$ is built up. It should be remarked that our curve (1) coincides with Lebesgue's curve within Cantor's set $T$ but not on the complement of $T$. 